\title{
Semi-empirical derived descriptors for the modelling of properties of $\mathrm{N}$-containing heterocycles
}

\author{
Alexander Entzian ${ }^{1 *}$, Horst Bögel ${ }^{1}$, Mirko Buchholz ${ }^{2}$, Ulrich Heiser ${ }^{2}$ \\ From 5th German Conference on Cheminformatics: 23. CIC-Workshop \\ Goslar, Germany. 8-10 November 2009
}

Nitrogen is one of the most prominent hetero atoms found in heterocycles. The corresponding electron lone pairs of these nitrogen atoms are mainly responsible for properties like the basicity and the pkb-values of the investigated heterocycle. Nevertheless, the overall electronic state of a molecule is also directly related to observable physico-chemical properties. This fact underlines a possible connection between different investigated properties.

The electronic properties of nitrogen containing compounds were analyzed with the aim to further predict these properties from quantum-mechanical descriptors. We thereby expect a relationship between the proton affinity, the pkb-value and the strength of metal complexation.

Because basicity seems to be a fundamental property of these compounds, the work was first focused on the proton affinities of the molecules in the gas phase. We thereby consider the fact, that these values are not influenced by the solvation in liquid phases. Lone pairs in nitrogen containing heterocycles play also an important role for metal complexation or due to their nucleophilic attack in chemical reactions. Therefore 55 heterocyclic compounds were selected that belong to the compound classes of substituted pyridines, pyrazoles and imidazoles. These compound set was carefully divided into a training (37) and test set (18), and different descriptors of the electronic states were calculated by using the semi-empirical molecular orbital software MSINDO [1].
In regression analysis the following descriptors were identified and marked as important:

- Charge of the nitrogen atom,

- Energy of the lone pair electrons and

- Perturbation treatment of the interaction energy Klopman [2].

Using this set of descriptors a model was built and validated that predicts the experimental data for proton affinity with an $\mathrm{R}^{2}$ of 0.93 and a $\mathrm{Q}^{2}$ of 0.91 . Furthermore, it was possible to successfully develop models for the prediction of pkb-values and the strength of a metal complex formation for these compounds.

\section{Author details}

${ }^{1}$ Martin-Luther-University Halle-Wittenberg, Institute for Organic Chemistry, Kurt-Mothes-Str. 2, 06120 Halle, Germany. ${ }^{2}$ Probiodrug AG, Dept. Medicinal Chemistry, Weinbergweg 22, 06120 Halle, Germany.

Published: 4 May 2010

\section{References}

1. Bredow T, Geudtner G, Jug K: J Comput Chem 2001, 22:861.

2. Klopman G: J Amer Chem Soc 1968, 90:223.

doi:10.1186/1758-2946-2-S1-P44

Cite this article as: Entzian et al:: Semi-empirical derived descriptors for the modelling of properties of $\mathrm{N}$-containing heterocycles. Journal of Cheminformatics 2010 2(Suppl 1):P44. 